\title{
Effect of microstructure closed-pore content on the mechanical properties of flexible polyurethane foam
}

\author{
Mohammad S. Gholamia,*, Olivier Doutres ${ }^{\mathrm{b}}$, Noureddine Atalla ${ }^{\mathrm{a}}$ \\ ${ }^{a}$ GAUS, Department of Mechanical Engineering, Université de Sherbrooke, Québec J1K \\ 2R1, Canada \\ ${ }^{b}$ Department of Mechanical Engineering, École de technologie supérieure (ÉTS), \\ Montreal H3C 1 K3, Canada
}

\begin{abstract}
Poroelastic models are constructed based on a set of physical parameters known as the Biot's parameters (for isotropic materials these are comprised of 5 non-acoustical parameters and 4 mechanical parameters). These macroscopic properties are inter-correlated and dependent on the microstructure of the foam. Therefore, to optimize vibroacoustic behavior, the correlation between foam microstructure and macroscopic properties is needed. In this study, the effect of closed windows content (known as reticulation rate), cell size, and relative density on the mechanical properties of polyurethane (PU) foam are numerically studied using an isotropic, tetrakaidecahedral unit cell. Then, an existing analytical model that correlates unit cell microstructure properties (thickness and length of struts) of fully reticulated foams to their mechanical properties is extended numerically. The membrane thickness is considered to be thinner at the center and larger at the edge of the window.
\end{abstract}

\footnotetext{
*Author to whom correspondence should be addressed.

Email address: mohammad.sadegh.gholami@usherbrooke.ca (Mohammad S. Gholami)
}

International Journal of Solids and Structures 112 (2017) 97-105, preprint version 
The ratio of membrane thickness to strut thickness is either considered to be fixed or variable. Results show that the Young's modulus and Poisson's ratio of unit cells with smaller cells size, when relative density is fixed, are more affected by closed window content.

Keywords: Polyurethane foam, Mechanical properties, poroelastic, Biot's parameter, Micro-Macro model, Reticulation rate.

\section{Introduction}

The skeleton of porous materials can be modeled as elastic, rigid, or limp (Allard and Atalla, 2009). Propagation of waves in elastic porous media, e.g. polymeric foams, is described by Biot-Allard's theory Allard and Atalla, 2009; Biot, 1956a b). Porous media with rigid frames (e.g. metal foams) and limp frames (e.g. soft fibrous) can be modeled using the same theory under specific assumptions which lead to simplified equations Allard and Atalla, 2009). Two classes of characteristic parameters are needed to describe the porous media in Biot-Allard's model. First, non-acoustic parameters: porosity $\phi$, thermal characteristic length $\Lambda^{\prime}$, viscous characteristic length $\Lambda$, flow resistivity $\sigma$, and tortuosity $\alpha$ which are used in JohnsonChampoux-Allard's (JCA) semi-phenomenological model Allard and Atalla, 2009; Champoux and Allard, 1991; Johnson et al., 1987). Second, mechanical parameters, in the case of isotropic material, are: bulk density $\rho$, Young's modulus $E$, loss factor $\eta$, and Poisson ratio $\nu$. It is well known that the mechanical and non-acoustical properties are inherently dependent on the microstructure properties of porous media. Hence, a clear understanding of the correlation between the microstructure and acoustical behavior of the 
foam is of utmost importance in the design and optimization of such foams. So far, the correlation between microstructure and non-acoustical parameters of foams with different internal structures has been studied by four different approaches: analytical (Allard and Atalla, 2009), scaling laws (Allard and Atalla, 2009; Doutres et al., 2011, 2013; Gibson and Ashby, 1999; Göransson, 2006; Perrot et al., 2007b), numerical (Hoang et al., 2014; Perrot et al., 2008, 2007a; Redenbach et al., 2012, Roberts and Garboczi, 2001), and empirical methods Cummings and Beadle, 1994; Kino and Ueno, 2008; Jang et al., 2015).

The internal structure of most porous material, such as highly porous polyurethane foam, is too complicated to be studied quantitatively. Therefore, a representative volume element (RVE) which is the minimum volume portion of a lattice containing the microstructure of the lattice and representing the behavior of the lattice, is considered (Li et al., 2003; Thiyagasundaram et al., 2010). The RVE in idealized PU foam is a tetrakaidecahedral unit cell which is periodic in a lattice. The microstructure properties of PU unit cell are: geometrical properties of strut (strut cross section shape and material distribution along the strut), thickness $t$ at the center of struts, length of struts $l$, reticulation rate $R_{w}$ (defined by (Doutres et al., 2011) as the ratio of the area of the open windows to the area of all windows which are placed on the same plane), cell size $C_{s}$, and relative density $\rho_{r}$ ( that is defined as the ratio of unit cell density to cell constituent material density $\rho / \rho_{s}$, and it is related to porosity with $\left.\phi=1-\rho_{r}\right)$.

The microstructural properties of tetrakaidecahedral unit cells are characterized using two-dimensional $(2 D)$ images obtained from a scanning electron 
microscope (SEM) (Doutres et al., 2013, 2011) (see Figure 1 a and b) or using three-dimensional $(3 D)$ images obtained from micro-computed tomography $(\mu C T)($ Jang et al., 2010; Perrot et al., 2007b; Veyhl et al., 2011) (see Figure 1. . Based on the morphology of PU foams, the microstructure of these foams with low relative density can be idealized by a lattice of repeating unit cells as shown in Figure 1d. Two kinds of Kelvin (after Thomson (1887)) unit cells are commonly considered in mechanical behavior studies (Gong et al., 2005; Hoang et al., 2014; Li et al., 2003). In this study, the tetrakaidecahedral unit cell, shown in Figure 11, is called KC1 and the other unit cell, Figure 1f, is named KC2. The correlation between the microstructure properties of foams with a tetrakaidecahedral unit cell shape and their mechanical properties has been studied by (Dawson and Shortall, 1982; Gong et al., 2005; Li et al., 2003; Mills, 2007; Shivakumar et al., 2011; Sullivan and Ghosn, 2009; Sullivan et al., 2008; Zhu et al., 1997). Previous studies can be mainly classified according to their assumptions on (1) deformation mechanisms (e.g. bending, axial, shear, or torsional deformation)

Zhu et al. (1997) presented analytically the correlation between the Young's modulus and relative density by considering that the deformation is caused by the bending and twisting in struts of tetrakaidecahedral unit cells. It was shown that the contribution of transverse shear forces and axial forces has the same order of magnitude as the effect of torsional deformation and accumulated mass at vertices (Li et al., 2003). Gong et al. (2005) analytically developed a micro-macro correlations for mechanical properties of fully reticulated PU foams considering all deformation mechanisms, non-uniformity in distributed mass along the strut with plateau border cross section, and the 

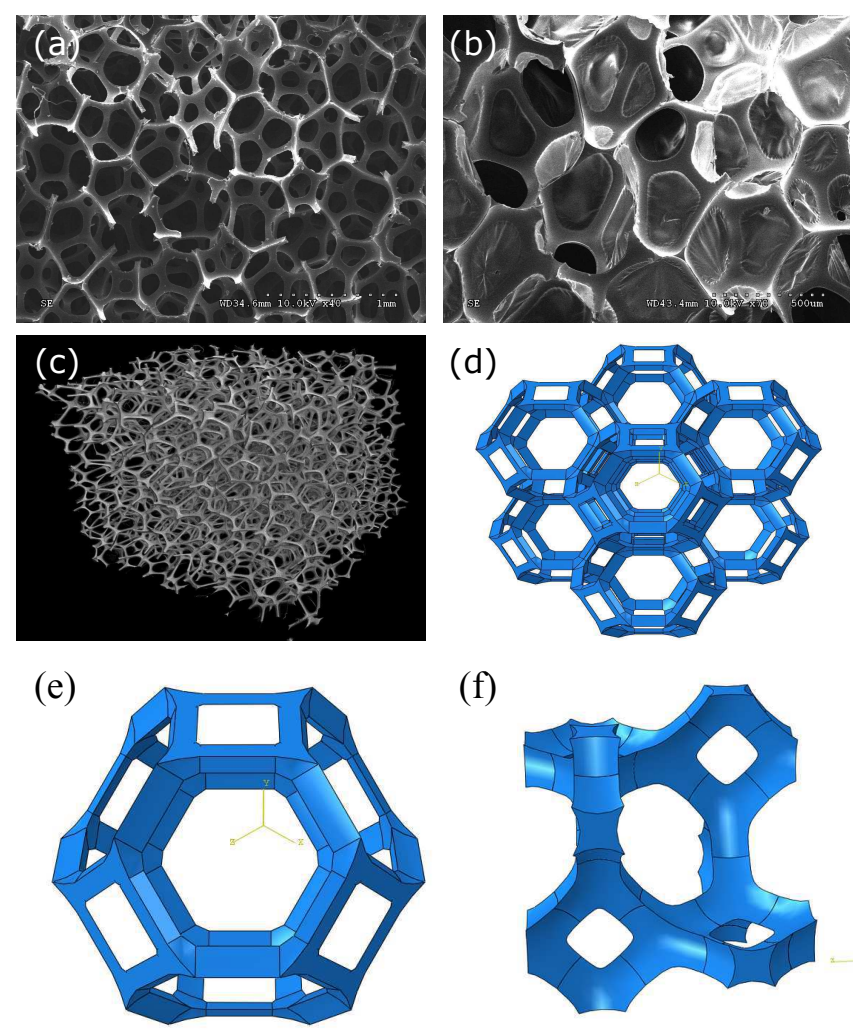

(f)

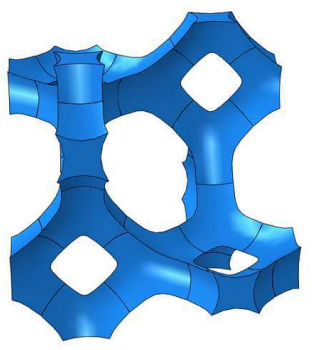

Figure 1: Lattice of PU foams (a) SEM photo $\left(R_{w}=100 \%\right)$. (b) SEM photo $\left(R_{w}=\right.$ 27\%)(c) X-Ray micro-tomography. (d) Idealized repeated unit cell. (e) KC1 (tetrakaidecahedral unit cell) (f) KC2. 
accumulated mass at the vertices. Two analytical models for unit cells with a uniform strut cross section and accumulated mass at the corners have been presented by Sullivan and Ghosn (2009) and Sullivan et al. (2008). In the former study, the deformation was because of axial and bending loads. While in a later study, shear effect was considered to be the only deformation mechanism. Later, Jang et al. (2008) modified the relations presented by Gong for struts with circular or triangular cross sections. This modified model is referred to by Gong and Jang's model in this study. Maheo et al. (2013) showed that the Young's modulus is more influenced by bending rather than shear, axial, and torsional deformation. An overestimation in predicted stiffness occurs by neglecting one or more deformation energies in the analytical model.

Analytical relations were found in good agreement with Finite Element (FE) modeling of an idealized unit cell presented (Gong et al., 2005; Jang et al., 2008; Li et al., 2003; Zhu et al., 1997). However, because of microstructure idealization, the analytical and the FE models were found to underestimate the Young's modulus when compared with experimental measurements (Gong et al., 2005; Jang et al., 2008). Therefore, empirical methods have been used to modify the relations based on idealization in geometry (Gong et al., 2005; Jang et al., 2008; Zhu et al., 1997). In some studies, the distribution of materials were considered to be uniform along the struts (Hoang et al., 2014; Li et al., 2003; Thiyagasundaram et al., 2010; Zhu et al., 1997), and in others, the accumulated mass at vertices was neglected (Li et al., 2003; Sullivan and Ghosn, 2009; Sullivan et al., 2008). Numerical methods were mainly used to investigate the effect of non-uniformity 
in cross sections, modified mass at vertices, or closed windows (Jang et al. 2008; Lu et al., 2011; Zhou et al., 2002). The non-uniformity in the strut cross sections thickness was found to have more impact on the elastic moduli than the shape of the cross sections (circle, triangle, and plateau border) and it was reported as the reason for increase in the Young's modulus (Gong et al., 2005; Jang et al., 2008; Kanaun and Tkachenko, 2007; Thiyagasundaram et al., 2010).

The mechanical models which are based on an idealized characteristic periodic unit cell (PUC) do not account for the cell irregularities that are typical of most foam structures. The influence of cell irregularity on mechanical properties of open-cell PU foam has been studied numerically by modeling the lattice (known as the Voronoi model) using the FE method (Gan et al., 2005; Jang et al., 2010, 2008; Kanaun and Tkachenko, 2007; Li et al., 2006; Zhu et al., 2000). To capture the behavior of the foam, at least 6 cells per edge are needed to model the lattice. This number increases to 21 cells per edge as irregularities increase. Modeling a Voronoi cell with a minimum of 6 cells per edge by using solid elements is difficult and computationally intensive. Therefore, a lattice was modeled by beam elements (Zhu et al., 2000). Reported results show that an increase in non-regularities results in an increase in the Young's modulus (Li et al., 2006, Zhu et al., 2000). It is worth mentioning that the influence of strut thickness non-uniformity is found less important than the effect of cell irregularity on the Young's modulus of the foam. Therefore, numerically periodic unit cell models are not as reliable as Voronoi models but are computationally less expensive.

Mechanical properties of either polymeric or metallic cellular materials 
with tetrakaidecahedral unit cell while all the windows are completely closed have also been studied (Redenbach et al., 2012; Jang et al., 2015; Chen et al., 2015; Andersons et al., 2016). Reduction in Young's modulus by increasing variability in cell size (Redenbach et al., 2012) and non-uniformity in cell wall thickness (Chen et al., 2015) are pointed out in the closed cell related numerical studies. In the latter study, it is assumed that at least $10 \%$ of windows remain open and unit cells stay interconnected through the open parts of windows. The closed window is known as a defect in the manufacturing process of open-cell AL foams. The impact of closed windows on mechanical properties of the Aluminum (AL) foams was analytically studied by using cubic cell models (Zhou et al., 2002) and numerically investigated for tetrakaidecahedral unit cells when the struts have a circular cross section (Jang et al. 2008, 2015; Lu et al., 2011). In these studies, windows of the unit cell are considered as either binary opened or closed (these windows are referred to here as binary reticulated). In Jang's and Lu's studies, it is assumed that the square windows have the same chance as hexagonal windows of being closed and the thickness of the closed windows is the same as the strut thickness. In addition, the relative density is kept the same as the open cell model (Lu et al., 2011). Therefore, less solid material is distributed to the struts of closed cells and, in consequence, the Young's modulus decreases as the percentage of closed windows increases. The Young's modulus of completely closed pore cellular material as a function of the relative density of the foam was studied by Jang et al. (2008), Lu et al. (2011), and Zhou et al. (2002).

The membrane impact on relative density of highly porous PU foams, 
the same PU foams used in this study, was shown negligible by Doutres et al. (2011). However, the relative density $\rho_{r}$ is affected by closed membrane content, when $\rho_{r} \geq 0.15$, in Robert's model Roberts and Garboczi, 2001). Elastic stiffness of four PU foams with different close pore content was reported by Zhang et al. (2012). Their results show that fully closed-pore, with $\rho_{r}=11.6 \%$, is $92.6 \%$ stiffer than fully-open windows PU foam. The effects of closed windows on the Young's modulus and the non-acoustical properties of two PU foams with different porosity were studied by Hoang et al. (2014). In their model, the foams' lattices are idealized by periodic unit cells. The struts have a circular cross section with uniform thickness along the length and spherical accumulated mass at the corners. The thickness of the membrane closing the windows is constant and the windows are partially reticulated. Solid elements are used to model the different unit cells including the membranes. The FE results were compared with experimental results obtained from static compression tests. To capture the mechanical properties of the foam, it was assumed that unit cells with cubic symmetry have random orientations for isotropic foam. A 164\% increase in Young's modulus, because of the presence of membrane, was reported for PU foams with $93 \%$ porosity, $9 \%$ reticulation rate, and $2 \mu m$ membrane thickness. This increase in the Young's modulus reaches 296\% when the thickness of the membrane is increased to $10 \mu \mathrm{m}$. The effect of the reticulation rate and membrane thickness on the Young's modulus of the foam with $97 \%$ porosity was reported as $768 \%$, and $1244 \%$ where the membrane thicknesses are $2 \mu \mathrm{m}$ and $10 \mu m$ respectively. In spite of these results, to the best of our knowledge, the impact of closed membrane content on Poisson's ratio and expressions 
describing the correlation between microstructure properties and mechanical properties haven’t been presented.

As mentioned in this section, the major number of previous micro-macro correlation studies on PU foams have been based on either fully reticulated or fully closed PU foam. The partially reticulated PU foam studies have not addressed a correlation to predict impact of closed pore content on mechanical properties of PU foam. In this study, not only the effect of reticulation rate but also cell size and relative density are numerically studied and used to extend an existing micro-macro model that correlates unit cell microstructure properties of fully reticulated foams to their mechanical properties. Hence, based on the range of relative density, cell size, and reticulation rate, obtained from microstructure characterization using SEM photos, different configurations of the $K C 1$ unit cell is modeled numerically in Section 2, The models are categorized based on two sets of relative densities, three different cell sizes, and six reticulation rates (an example of when relative density is fixed and different configurations can be created by a combination of different cell sizes and reticulation rates is shown in Figure 21. In section 3, the Young's modulus and Poisson's ratio are identified by performing a compression and a shear numerical test for each configuration. The modified micro-macro correlation are presented to add the impact of reticulation rate, cell size, and relative density into the Gong-Jang's analytical model. In Section 3.2, quasi static tests are used to corroborate the validity of the proposed expressions and show the effect of closed membrane on the Young's modulus of PU foams with different microstructure properties. 

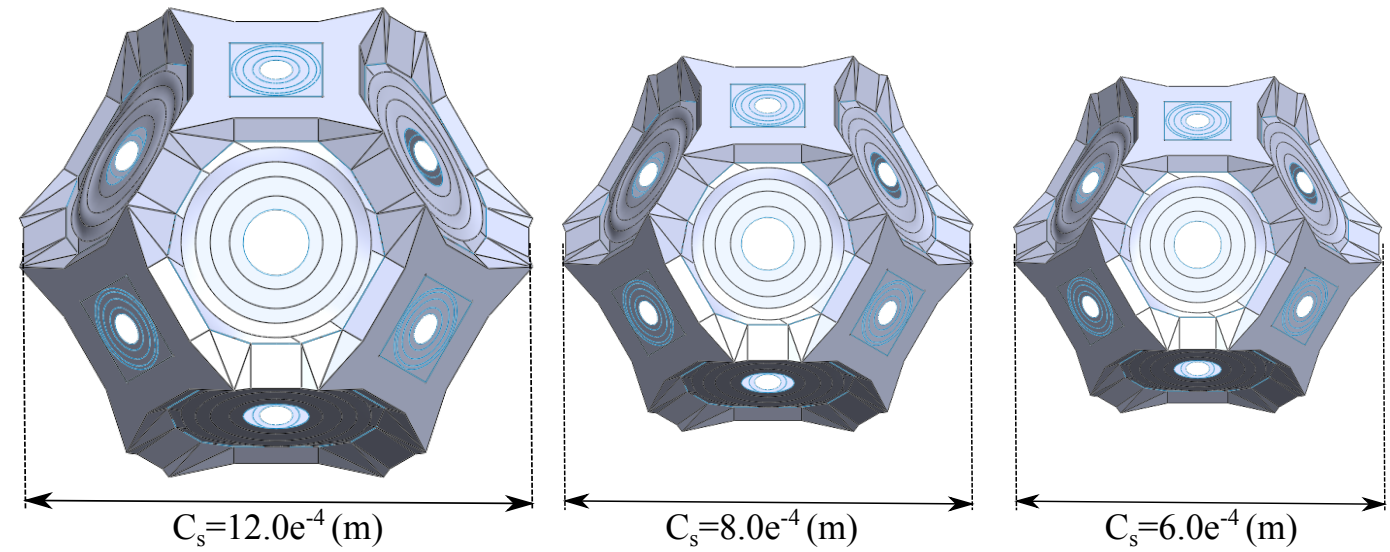

Figure 2: Unit cells with three cell sizes and six reticulation rates.

\section{Mechanical properties of Unit Cell}

\subsection{Numerical model for unit Cell}

Since $K C 1$ provides a higher number of windows (8 quadrilateral and 8 hexagon), it is preferred to study the impact of reticulation rate on mechanical properties of PU foam. This is of value for reticulated windows since a wider range for reticulation rate is reachable. In most cases, non-isotropic properties are expected since the cells are elongated in the rise direction due to the foaming and rising process. The focus of this work lies on the impact of the membrane closing the windows. Hence, the degree of anisotropy, which is defined as the ratio of the maximum height of cells to the lateral dimensions, is taken equal to one (Doutres et al., 2011; Gibson and Ashby, 1999; Ridha and Shim, 2008). The geometry of the unit cells are created in SOLIDWORKS-(Dassault Systèmes) and FE models are solved in Abaqus 6.14-2-(Dassault Systèmes). The quadratic tetrahedron (type C3D10) element is selected for struts and vertices. To impose the periodic boundary 


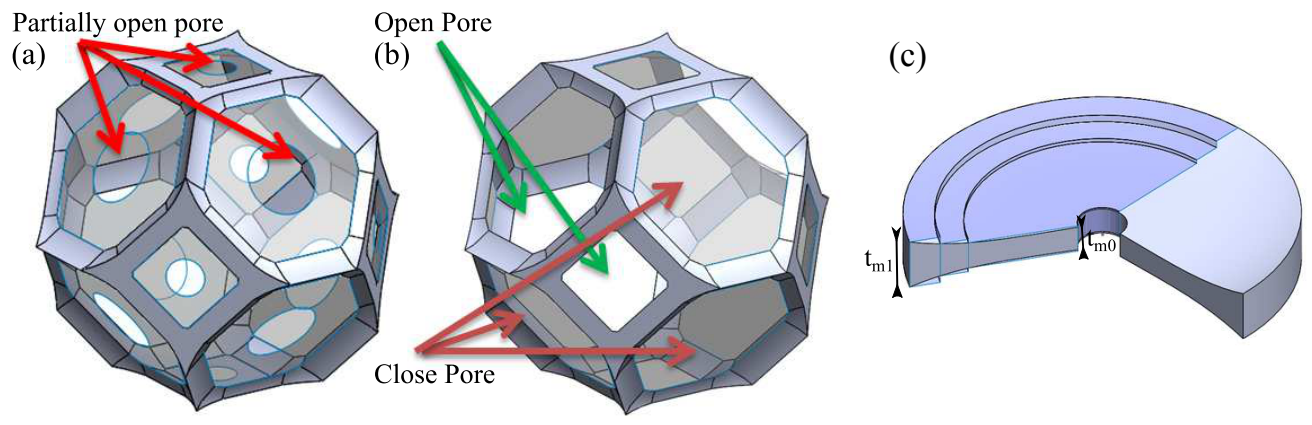

Figure 3: Methods used to create different reticulation rate. (a) Removing a fraction of the surface of all membranes (Hoang et al., 2014). (b) Pores are binary open or close (Doutres et al. 2013, 2011). (c) Variable membrane thickness is modeled using shell elements.

condition $(\mathrm{PBC})$, the mesh patterns on the left, bottom, and front sides are copied to the right, top, and rear sides of the unit cell respectively.

Reticulation rate should be defined as the ratio of the area of the open windows to the area of all windows when a 3-dimensional unit cell is used in numerical modeling. The pore of unit cell can be either proportionally reticulated by opening a ratio of the membrane or binary reticulated (the windows are fully opened or fully closed) as shown in Figure 3 a \& b respectively. In this study, the windows are proportionally reticulated (Figure 3 a) because the cubic symmetry condition is not valid when a unit cells carrying binary reticulated windows. The membrane thickness is assumed to be variable as shown in Figure 3 c. Shell elements (linear quadrilateral S4R) are used to assess the membrane effect. In the literature, the measured values for the constituent material (struts) mechanical properties are scattered over a wide range ([2 - 69] MPa) Gibson and Ashby, 1999; Gong et al., 2005 . Jang et al., 2008) due to measurement uncertainties. This study uses the mechanical properties measured by Gong et al. (2005) as listed in Table 1. 


\begin{tabular}{l|c}
\hline \hline Material properties of the constituent material(Gong et al., 2005$)$ & \\
\hline Density, $\rho_{s}, \mathrm{~kg} / \mathrm{m}^{3}$ & 1190 \\
Elastic modulus, $E_{s}, \mathrm{~Pa}$ & $69 e 6$ \\
Poisson's ratio, $\nu$, & 0.49 \\
\hline \hline
\end{tabular}

Table 1: Material properties of the struts

\subsection{Periodic boundary condition}

In the following, Young's modulus and Poisson's ratio of unit cells are numerically identified by a compression and a shear deformation test. Based on loading type, appropriate periodic boundary conditions are applied to the nodes placed on boundary surfaces of the unit cell. Displacement of coincidence nodes placed on parallel boundary surfaces in the compression test is written as (Xia et al., 2006):

$$
u_{i}^{+j}-u_{i}^{-j}=\bar{\epsilon}_{i k} \Delta x_{k}^{j}
$$

where $i, j=1,2,3$, and superscripts $+j$ and $-j$ stand for the $j$ th pair of two opposite parallel surfaces of the unit cell (in directions $+\&-$ ). $\Delta x_{k}^{j}=$ $\left(x_{k}^{+j}-x_{k}^{-j}\right)$ are constants for each pair of the parallel boundary surfaces. Therefore, the right side of the equation will be constant for specified $\overline{\boldsymbol{\epsilon}}_{i k}$. As an exapmle, the imposed PBC to the unit cells subjected to a normal 
compression test $\left(\overline{\boldsymbol{\epsilon}}_{22}\right)$ are:

$$
\begin{aligned}
& u_{i}^{(+1)}-u_{i}^{(-1)}=0, \\
& u_{i}^{(+3)}-u_{i}^{(-3)}=0 .
\end{aligned}
$$

for nodes belonging to the right, left, front, and rear faces. And, each pair of nodes placed on top $([0,1,0])$ and bottom $([0,-1,0])$ planes are constrained as:

$$
\begin{aligned}
& u_{1}^{(+2)}-u_{1}^{(-2)}=0, \\
& u_{2}^{(+2)}-u_{2}^{(-2)}=\overline{\boldsymbol{\epsilon}}_{22} C_{s}, \\
& u_{3}^{(+2)}-u_{3}^{(-2)}=0 .
\end{aligned}
$$

where $C_{s}$ is the cell size $\left(\Delta x_{2}^{2}\right)$. These constraints are imposed to the unit cells. The PBCs for the shear test are defined based on deformation gradients $\gamma_{i, j}=u_{i, j}+u_{j, i}$. Here, only $u_{i, j}$ is applied. Therefore, two sets of BCs are applied simultaneously as shown in the following (Thiyagasundaram et al. 2010):

$$
\begin{aligned}
\left(u_{i}^{+j}-u_{i}^{-j}\right) & =\bar{\gamma}_{i j} C_{s}, \\
\left(u_{j}^{+i}-u_{j}^{-i}\right) & =0 .
\end{aligned}
$$

where $\overline{\gamma_{i j}}$ is the shear strain. The periodic boundary conditions (Equations 1 and 4) are applied on boundary surfaces of the unit cell by using constraint equations in Abaqus. To do so, the boundary mesh pattern of a master surface is copied on the opposite boundary surface of the RVE (see Figure 4) and then, mesh is generated Storm et al. (2015). 


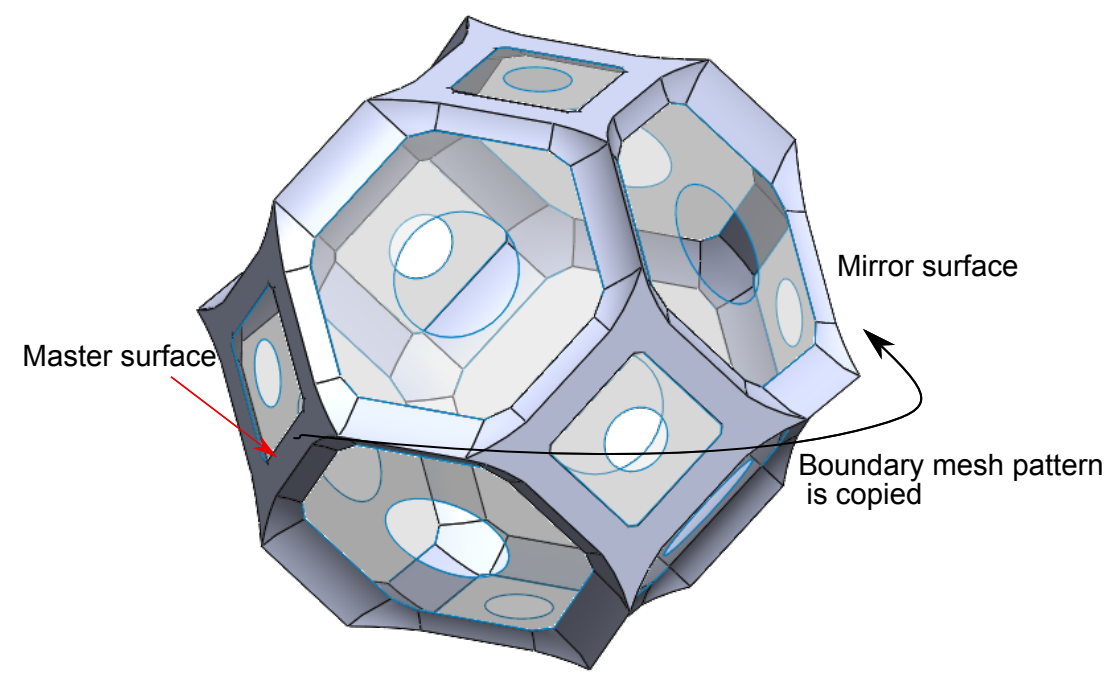

Figure 4: Boundary mesh pattern of a master surface is copied on the mirror surface.

\subsection{Derivation of the elastic constants}

In this section the procedure of deriving linear elastic properties of the unit cell is detailed. The tetrakaidecahedral unit cell $K C 1$ with partially reticulated membrane shows cubic symmetry. Therefore, the elastic tensor is defined by three independent elasticity components: $C_{11}, C_{12}$, and $C_{44}$.

$$
\left\{\begin{array}{l}
\overline{\boldsymbol{\sigma}}_{11} \\
\overline{\boldsymbol{\sigma}}_{22} \\
\overline{\boldsymbol{\sigma}}_{33} \\
\overline{\boldsymbol{\sigma}}_{23} \\
\overline{\boldsymbol{\sigma}}_{13} \\
\overline{\boldsymbol{\sigma}}_{12}
\end{array}\right\}=\left[\begin{array}{cccccc}
C_{11} & C_{12} & C_{12} & 0 & 0 & 0 \\
& C_{11} & C_{12} & 0 & 0 & 0 \\
& & C_{11} & 0 & 0 & 0 \\
& & & C_{44} & 0 & 0 \\
& & & & C_{44} & 0 \\
& & & & & C_{44}
\end{array}\right]\left\{\begin{array}{c}
\overline{\boldsymbol{\epsilon}}_{11} \\
\overline{\boldsymbol{\epsilon}}_{22} \\
\overline{\boldsymbol{\epsilon}}_{33} \\
2 \overline{\boldsymbol{\epsilon}}_{23} \\
2 \overline{\boldsymbol{\epsilon}}_{13} \\
2 \overline{\boldsymbol{\epsilon}}_{12}
\end{array}\right\},
$$

the elasticity tensor component are: $C_{11}=\lambda+2 \mu, C_{12}=\lambda$, and $C_{44}=$ 
$\mu$. where, $\lambda$ and $\mu$ are Lamé constants. Therefore, $C_{44}=\mu$ is derived using a shear test together with associated periodicity conditions presented in Equation 4 and a compression test (PBCs defined in Equation 1) results in longitudinal modulus $C_{11}$, and $C_{12}$. Mechanical properties are calculated as shown in the following:

$$
\begin{array}{r}
\lambda=\frac{E \nu}{(1+\nu)(1-2 \nu)}, \\
\mu=G=\frac{E}{2(1+\nu)} .
\end{array}
$$

\section{Results}

\subsection{Impact of closed-windows on the mechanical properties}

Before adding membrane into unit cells, mechanical properties of a fully reticulated $R_{w}=100 \%$ unit cell obtained from numerical calculations are val-

idated by comparison with the Gong-Jang's analytical models (Equations 7 \& 8). It should be mentioned that Equations 7 and 8 are simplified for the case unit cell is isotropic.

$$
\begin{gathered}
\frac{E}{E_{s}}=\frac{\left(\frac{\sqrt{2}}{2}\right) C_{I} C_{A}^{2}\left(\frac{t}{l}\right)^{4}}{C_{1}+2 C_{I} C_{A} C_{2}(0.5+\beta(1+\nu))\left(\frac{t}{l}\right)^{2}}, \\
\nu=\frac{1}{2}\left(\frac{C_{1}-C_{I} C_{A} C_{2}\left(\frac{t}{l}\right)^{2}}{C_{1}+C_{I} C_{A} C_{2}\left(\frac{t}{l}\right)^{2}}\right)
\end{gathered}
$$

where $C_{I}=0.1338, C_{A}=\sqrt{(3)}-\pi / 2$, and $\beta=1.24$ are geometrical constants for plateau border cross section shape of the struts. $C_{1}=0.0168241$ and $C_{2}=0.654375$ are dependent on the distributed material along the strut 
thickness. They are explained in the Gong and Jang's study (Gong et al., 2005; Jang et al., 2008). As shown in Figure 5 a \& b, SEM photos are used to measure the distribution of material along the strut, strut length (Figure 5 c), and strut thickness (Figure 5 d).

Indeed, the micro-macro models presented in Equations 7 and 8 depend on microstructure properties of Gong and Jang's model (ratio of strut length to thickness, strut cross section shape, and non-uniformity of strut thickness). Morphology of the studied PU foams, using SEM photos, shows high variability in the ratio of strut length to strut thickness. The variability is associated with anisotropy which is a consequence of elongation in the rise direction during the foaming process. Wide normal distribution for $l / t$ results in divers prediction of Young's modulus. Hence, the mean value of $l / t$ of each PU foam, before being used in either Equation 7 or Equation 8 , is validated through comparison of the porosity estimated using Equation 9 and experimental measurements. Therefore, the model presented by Doutres et al. (2011) is recalled here:

$$
\phi=1-\rho_{r}=1-\frac{2 \sqrt{3}-\pi}{\sqrt{2}}\left(\frac{t}{l}\right)^{2}
$$

A fully reticulated unit cell when cell size is fixed to $C_{s}=600 \times 10^{-6}(\mathrm{~m})$ and $l / t=3.0$, that results in $\rho_{r}=2.6 \%$, is considered to compare the numerical and analytical predictions. Properties of the constituent strut material of unit cells are listed in Table 1. Results show that numerical model $\left(E / E_{s}=0.14 \%\right.$ and $\left.\nu=0.43\right)$ captures accurately the predicted result obtained from analytical Gong-Jang's model $\left(E / E_{s}=0.13 \%\right.$ and $\left.\nu=0.43\right)$. The reasons for small differences between the analytical model and FE models can be attributed to limitations in CAD models to create the exact profile 

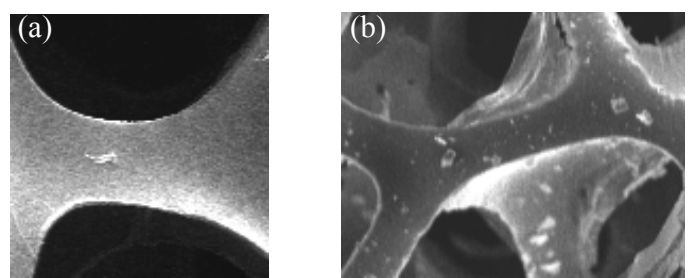

(c)

(d)

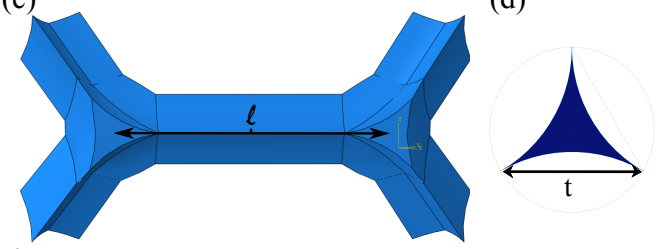

Figure 5: The SEM photos of the struts to show the variation of cross section area of the strut in studied PU foams. (a) PU1, (b) PU14, (c) length of strut used in FE model, and (d) strut thickness at the center of strut.

for struts, removing the exact amount of material at the corners in numerical models, and difficulties in determining deformation energies at the vertices in the analytical model. In the following, the numerical model will be modified in order to account for the presence of membranes closing the pores and its results used to propose an augmented Gong-Jang's model (Equations 7 and 8).

The tetrakaidecahedral unit cells $K C 1$ with different reticulation rate, cell size, and relative densities are considered to identify the effect of cell properties on mechanical properties of a lattice of PU foam. It is worth mentioning that different reticulation rates are provided numerically by closing a fraction of windows as shown in Figure 3 a. In the first set of numerical calculations, the membrane thickness is fixed in all models. Therefore, the ratio of membrane thickness to the strut thickness is reduced by increasing the cell size (relative density is fixed $l / t=$ constant, therefore thickness of strut 
$t$ increases as cell size increases, $C_{s}=2 l \sqrt{2}$ ). Measurements show a slight increase in membrane thickness at struts edge vicinity. Membrane thicknesses at the center and near the strut edges of a studied PU foam (PU5) are measured using SEM photos as $t_{m 0}=2.1 \pm 0.45(\mu m)$ and $t_{m 1}=3.0 \pm 0.40(\mu m)$ respectively. Therefore, the nominal values of the membrane thicknesses are considered in the first set of numerical models. The associated results are shown in Figure 6.

The Young's modulus and Poisson's ratio are numerically calculated using the approach of Section 2.3. Figure 6 shows the impact of variation in unit cell properties on mechanical properties of the PU foams for the first set of calculations. In Figure $6, E_{100}\left(\nu_{100}\right)$ stands for the Young's modulus (Poisson's ratio) of fully reticulated PU foam, $E_{R_{w}}\left(\nu_{R_{w}}\right)$ is the Young's modulus (Poisson's ratio) of partially reticulated PU foam, and vertical axis presents the impact of adding membrane on mechanical properties. The unit cells relative density are fixed to $\rho_{r}=2.6 \%$ in Figures 6 a \& c $($ or $l / t=3.0)$, while Figures $6 \mathrm{~b} \& \mathrm{~d}$ present the results when $\rho_{r}=3.6 \%(l / t=2.6)$. As shown in Figures 6, the impact of reticulation rate on mechanical properties of PU foam increases as cell size decreases. In other words, for a fixed relative density, cell size reduction results in decrease in strut thickness. Therefore, the ratio of membrane thickness to strut thickness increases. The same trend can be observed for relative density. The maximum reticulation rate effect on Young's modulus and Poisson's ratio, when unit cell size and reticulation rate are minimum, reach $300 \%$ and $25.0 \%$ respectively. It is also shown in Figure 6b that, for relative density $\rho_{r}=3.6 \%$, the cell size impact on mechanical properties of the foam when reticulation rate is larger than $85 \%$ is 
very low. Comparing mechanical properties at fully reticulated points $\left(R_{w}=\right.$ $100 \%$ ) indicates that, as long as relative density remains constant, mechanical properties of unit cell are independent from cell size. Computational results point out that the impact of closing windows on the Young's modulus is additive while this effect on Poisson's ratio is subtractive. This means that adding membrane to the windows results in a higher Young's modulus and lower Poisson's ratio in comparison with fully opened windows.

Using results presented in Figure 6, the following two relations are proposed to predict the impact of cell microstructure properties on its mechanical properties.

$$
\begin{aligned}
\frac{\Delta E}{E_{100}}= & 6.6263 e-4\left(\frac{1}{C_{s}}\right)^{0.55}\left(\frac{1}{\rho_{r}}\right) R w^{2}-1.8104 e \\
& -4\left(\frac{1}{C_{s}}\right)^{0.65}\left(\frac{1}{\rho_{r}}\right)^{1.5} R w+2.4982 e-5\left(\frac{1}{C_{s}}\right)^{0.75}\left(\frac{1}{\rho_{r}}\right)^{1.75}, \\
\frac{\Delta \nu}{\nu_{100}}= & -\left(0.0122\left(\frac{1}{C_{s}}\right)^{0.25}\left(\frac{1}{\rho_{r}}\right)^{0.2} R_{w}^{2}\right. \\
& \left.-0.0123\left(\frac{1}{C_{s}}\right)^{0.35}\left(\frac{1}{\rho_{r}}\right)^{0.3} R w+0.0027\left(\frac{1}{C_{s}}\right)^{0.45}\left(\frac{1}{\rho_{r}}\right)^{0.4}\right) .
\end{aligned}
$$

where, $\Delta E=\left(E_{R_{w}}-E_{100}\right), \Delta \nu=\left(\nu_{R_{w}}-\nu_{100}\right)$, reticulation rate $R_{w}$ can be $10 \leq R_{w}(\%) \leq 100$, and $C_{s}$ is cell size $(\mathrm{m})$. The predicted difference (dashed lines in Figure 6) show that Equations 10 \& 11 capture the FEM results. Relative density is related to strut length and thickness as mentioned in Equation 9. The cell size is equal to $C_{s}=A l \sqrt{2}$, where $A=2$ for isotropic unit cell. For PU foams considered in this paper, Doutres et al. (2013) found constant $A=2.33 \pm 0.36$ very close to the isotropic unit cell. The relative 

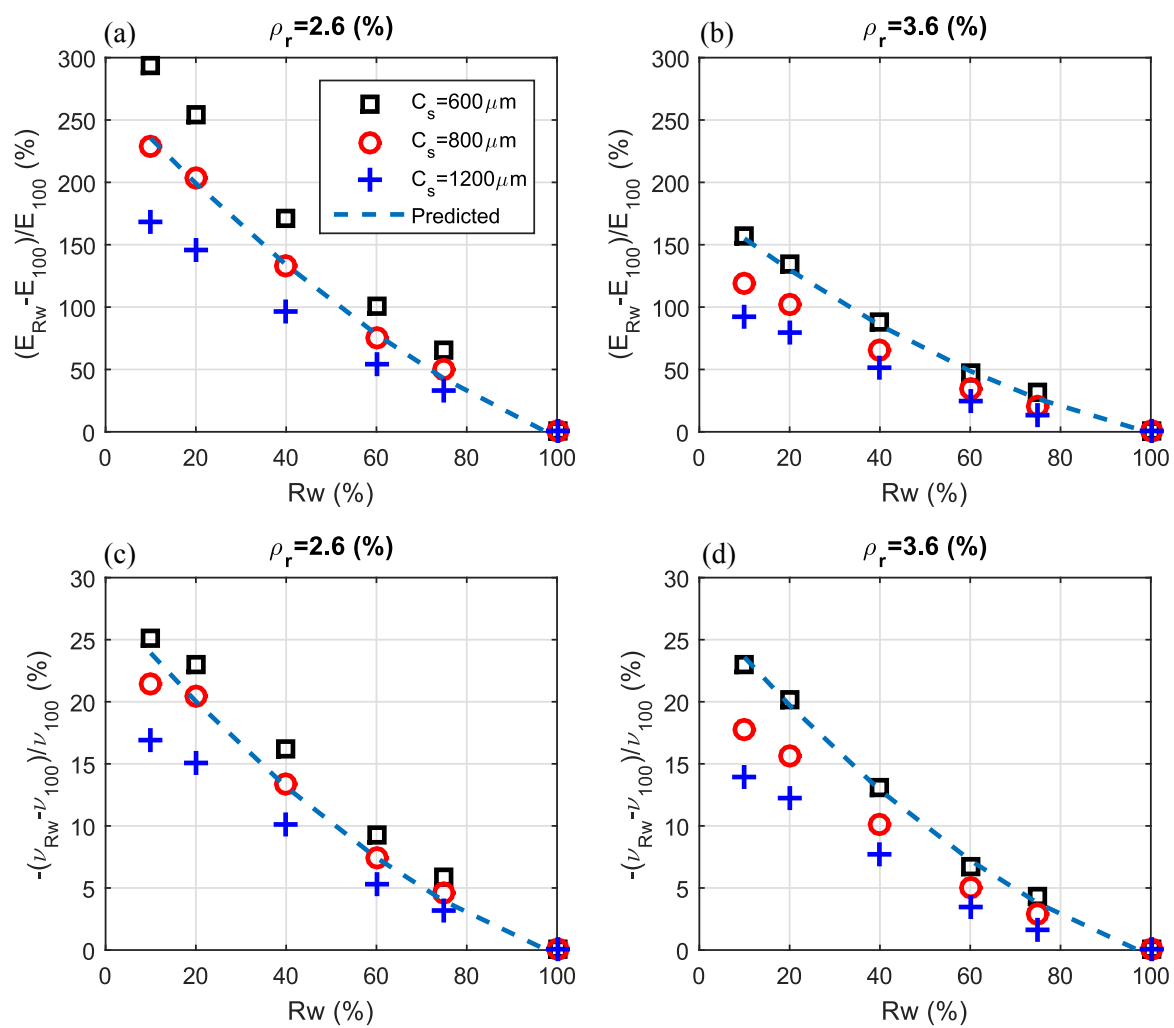

Figure 6: The impact of reticulation rate on mechanical properties of PU foam for different cell sizes and relative densities. Relative density for (a) and (c) is $\rho_{r}=2.6 \%$ and for (b) and (d) is $\rho_{r}=3.6 \%$. (a) and (b) present the impact of closed windows content on Young's modulus while (c) and (d) show the impact of reticulation rate on Poisson's ratio. The dashed line shows predicted results using Equations 10 \& 11. 


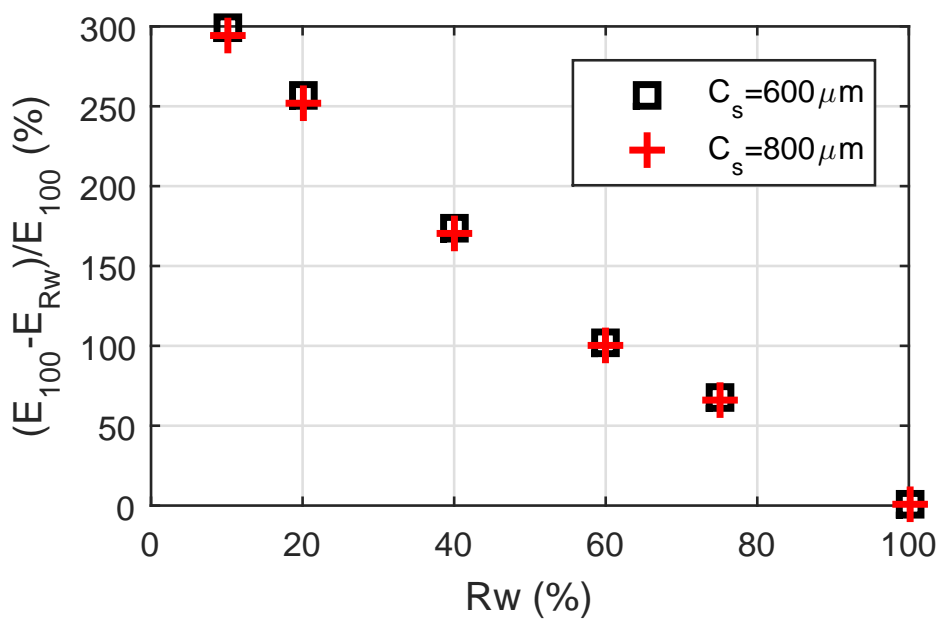

Figure 7: The ratio of membrane thickness to the strut length remains constant for two cells with different cell sizes and fixed ratio of $l / t$.

Young's modulus and Poisson's ratio of partially reticulated PU foam are calculated using Equation 10 and Equation 11. $\quad E_{100}$ and $\nu_{100}$ calculated using Equations 7 and 8 respectively.

In the second set of numerical calculations, the ratio of membrane thickness to strut thickness is fixed and it is assumed to be the same as PU5 that is $4.2 \%$ at edges and $3.1 \%$ at center. Figure 7 shows the results when the ratio of membrane thickness to strut thickness remains unchanged but cell size varies. As depicted here, for cells with the same relative density, the cell size impact vanishes when ratio of membrane thickness to strut thickness is kept constant. It should be mentioned that the ratio $(l / t)$ is fixed. 


\subsection{Validation}

\subsubsection{Experimental setup:}

Impact of the reticulation rate on the mechanical properties of PU foams is investigated experimentally by performing compression tests at low-frequency using a quasi-static method (QMA) (Langlois et al., 2001). In this method, at least two cylindrical samples with different shape factors (the ratio of half radius to thickness of the sample) are used. The samples are placed between two rigid plates with non-slippery surface conditions. A small amplitude, sinusoidal compression is imposed and the reaction force is measured. Based on the method proposed by Langlois et al. (2001), the Young's modulus and the Poisson's ratio are measured. To avoid non-linearity effects, a maximum static strain of $2 \%$ is applied to the samples while dynamic strain is $0.02 \%$. The Young's modulus of 20 PU foams is measured using the QMA method and results are listed in Table 2, Furthermore, reticulation rate $R_{w}$, cell size $C_{s}$, and ratio of $l / t$ are characterized using SEM micrograph. Porosity $\phi$ is measured using the method presented by Salissou and Panneton (2007).

\subsubsection{Comparison of experimental and numerical results}

Results obtained from numerical investigations are compared with experimental measurements. To do so, twenty PU foams, listed in Table 2, are classified into four groups based on the cell size. Group A represents PU foams with cell size within $C_{s}=655 \pm 58(\mu \mathrm{m})$. Group B includes foams with $C_{s}=761 \pm 78(\mu m)$, Group $\mathrm{C}$ foams with $C_{s}=1247 \pm 103(\mu m)$, and Group D foams with $C_{s}=1562 \pm 124(\mu m)$. The first column in Table 2 indicates the group that each foam belongs to. Experimental measurements corroborate the numerical results in such a way that reticulation rate impact 


\begin{tabular}{l|c|c|c|c|c|c}
\hline \hline Group & Foam & $R_{w}(\%)$ & Cell size $(\mu m)$ & $l / t$ & $\phi(\%)$ & $E_{Q M A}(k P a)$ \\
\hline A & PU1 & 100 & $680 \pm 90$ & $3.2 \pm 1.2$ & $95.1 \pm 1.8$ & $250 \pm 10$ \\
A & PU2 & 100 & $650 \pm 40$ & $2.8 \pm 0.9$ & $97.2 \pm 1.8$ & $274 \pm 20$ \\
A & PU10 & 100 & $671 \pm 80$ & $3.2 \pm 0.9$ & $96.8 \pm 1.8$ & $301 \pm 14$ \\
A & PU11 & 100 & $665 \pm 40$ & $2.5 \pm 0.9$ & $95.9 \pm 1.8$ & $221 \pm 10$ \\
A & PU16 & 100 & $540 \pm 40$ & $3.2 \pm 1.3$ & $95.5 \pm 1.8$ & $248 \pm 11$ \\
A & PU14 & 100 & $689 \pm 35$ & $3.3 \pm 1.0$ & $96.4 \pm 1.7$ & $229 \pm 12$ \\
A & PU12 & 86 & $681 \pm 50$ & $2.8 \pm 1.0$ & $94.8 \pm 1.7$ & $282 \pm 22$ \\
A & PU7 & 27 & $671 \pm 68$ & $3.1 \pm 1.2$ & $96.7 \pm 1.8$ & $465 \pm 25$ \\
B & PU6 & 100 & $750 \pm 80$ & $2.8 \pm 1.0$ & $96.1 \pm 1.8$ & $240 \pm 15$ \\
B & PU15 & 100 & $727 \pm 84$ & $3.2 \pm 1.0$ & $95.1 \pm 1.8$ & $223 \pm 14$ \\
B & PU5 & 38 & $730 \pm 51$ & $2.9 \pm 0.9$ & $95.6 \pm 1.8$ & $295 \pm 18$ \\
B & PU18 & 28 & $878 \pm 93$ & $3.0 \pm 1.0$ & $97.4 \pm 1.7$ & $313 \pm 13$ \\
B & PU13 & 20 & $722 \pm 80$ & $2.6 \pm 0.9$ & $94.4 \pm 1.6$ & $368 \pm 40$ \\
C & PU19 & 100 & $1190 \pm 122$ & $3.4 \pm 1$ & $96.4 \pm 1.8$ & $235 \pm 18$ \\
C & PU20 & 100 & $1352 \pm 63$ & $3.0 \pm 1$ & $96.1 \pm 1.8$ & $227 \pm 12$ \\
C & PU17 & 11 & $1200 \pm 115$ & $2.7 \pm 1.1$ & $96.3 \pm 1.8$ & $323 \pm 32$ \\
D & PU8 & 100 & $1450 \pm 150$ & $2.8 \pm 0.8$ & $97.0 \pm 1.8$ & $284 \pm 19$ \\
D & PU9 & 100 & $1350 \pm 150$ & $3.2 \pm 1.0$ & $96.4 \pm 1.2$ & $260 \pm 05$ \\
D & PU3 & 30 & $1700 \pm 100$ & $3.8 \pm 1.2$ & $94.5 \pm 1.9$ & $272 \pm 18$ \\
D & PU4 & 31 & $1750 \pm 100$ & $3.3 \pm 1.1$ & $94.9 \pm 1.7$ & $274 \pm 17$ \\
\hline \hline
\end{tabular}

Table 2: Reticulation rate, PUC properties, and Young's modulus of PU foams. 
on the Young's modulus has increased by reducing the reticulation rate. In addition, the impact of closed pore content decreases when cell size increases. To compare with Equation 10, cell size, relative density, and reticulation rate are needed. An average relative density and cell size is thus used for every set of foams in Equation 10. It should be added that the maximum ratio of membrane to strut thickness is $4 \%$ when membrane thickness is $t m 1=3 \mu m$ and strut thickness is $t=70 \mu m$. Results show that, apart from foam in group D, Equation 10 is able to capture the effect of added membrane on the Young's modulus. However, the difference is related to the uncertainty and variability in microstructure properties of studied PU foams and experimental measurements of Young's modulus. Measurement of membrane thickness and its constituent material properties are added to the possible origins of discrepancies when PU foams are partially reticulated. The predicted Young's modulus of foams in Groups A, B, and C are commonly overestimated. This can be explained as unit cell idealization results in more flexible representative unit cell structures. Therefore, membrane closing the windows plays a more important role in the stiffness of the unit cell. In addition, uncertainties on constituent material and experimental measurement such as imposing static compression using the QMA method affects the Young's modulus Geslain et al., 2011) are other reasons for difference in prediction and experimental measurements. It should be mentioned that, because of a lack of accuracy in the used experimental measurements, results of experimental measurements of Poisson's ratio have not been reported here. 


\section{Conclusion}

The Biot's parameters are inter-correlated and are microstructure-dependent. There is, in consequence, a need for the development of links between the cellular structure of the foams and the Biot's parameters before realistically using these models for material-level optimization. In this sense, a microstructure-based model has been developed by Doutres et al. (2013, 2011) to link the microstructure (thickness and length of struts and the closed windows content) of polyurethane foams to their non-acoustical parameters. An analytical relation which correlates the macroscopic mechanical properties of fully reticulated PU foams into their microstructure parameters has been presented by Gong et al. (2005) and Jang et al. (2008). In this study, the latter model is augmented numerically to account for the effect of reticulation rate, cell size, and relative density on the mechanical properties of PU foams. The membrane thickness is considered to be variable. Results show that the impact of closed pore content on mechanical properties of the PU foam reduces by either increasing the cell size or relative density of the foam. In addition, the Young's modulus of the foam increases by closing a greater portion of the windows while the Poisson's ratio decreases for the same amount of variation in closed pore content. Anisotropy in PU foams results in an increase in variability in ratio of the strut thickness to the struts length. Therefore, the ratio of struts thickness to the length should be averaged over all struts using SEM photos. Micro-structure properties and Young's modulus of 20 PU foams are characterized using SEM photos and QMA measurement respectively. Results show that the predicted effect of reticulation rate on the Young's modulus is qualitatively in accordance with 
the measured Young's modulus.

\section{ACKNOWLEDGMENT}

The authors would like to thank the Natural Sciences and Engineering Research Council of Canada (NSERC) for providing financial support.

\section{References}

Allard, J., Atalla, N., 2009. Propagation of Sound in Porous Media: Modelling Sound Absorbing Materials, 2nd Edition. WILEY.

Andersons, J., Kirpluks, M., Stiebra, L., Cabulis, U., 2016. Anisotropy of the stiffness and strength of rigid low-density closed-cell polyisocyanurate foams. Materials and Design 92, 836-845.

URL http://dx.doi.org/10.1016/j.matdes.2015.12.122

Biot, M. A., 1956a. Theory of Propagation of Elastic Waves in a FluidSaturated Porous Solid. II. Higher Frequency Range.

Biot, M. A., 1956b. Theory of propagation of elastic waves Low frequency range. The Journal of the Acoustical Society of America 168 (1956), $179-191$.

URL http://scitation.aip.org/content/asa/journal/jasa/28/ 2/10.1121/1.1908241http://scitation.aip.org/content/asa/ journal/jasa/28/2/10.1121/1.1908239

Champoux, Y., Allard, J.-F., 1991. Dynamic tortuosity and bulk modulus in air-saturated porous media. Journal of Applied Physics 70 (4), 1975-1979. 
URL http://scitation.aip.org/content/aip/journal/jap/70/4/ $10.1063 / 1.349482$

Chen, Y., Das, R., Battley, M., 2015. Effects of cell size and cell wall thickness variations on the stiffness of closed-cell foams. International Journal of Solids and Structures 52, 150-164.

URL http://dx.doi.org/10.1016/j.ijsolstr.2014.09.022

Cummings, A., Beadle, S., 1994. Acoustic properties of reticulated plastic foams. Journal of sound and vibration 175 (1), 115-133.

URL http://wWw.sciencedirect.com/science/article/pii/ S0022460X84713150

Dawson, J. R., Shortall, J. B., jan 1982. The microstructure of rigid polyurethane foams. Journal of Materials Science 17 (1), 220-224.

URL http://link.springer.com/10.1007/BF00809056

Doutres, O., Atalla, N., Dong, K., 2011. Effect of the microstructure closed pore content on the acoustic behavior of polyurethane foams. Journal of Applied Physics 110 (6), 064901.

URL http://link.aip.org/link/JAPIAU/v110/i6/p064901/ s1\{\&\}Agg=doi

Doutres, O., Atalla, N., Dong, K., 2013. A semi-phenomenological model to predict the acoustic behavior of fully and partially reticulated polyurethane foams. Journal of Applied Physics 113 (5), 054901.

URL http://link.aip.org/link/JAPIAU/v113/i5/p054901/ s1\{\&\}Agg=doi 
Gan, Y., Chen, C., Shen, Y., dec 2005. Three-dimensional modeling of the mechanical property of linearly elastic open cell foams. International Journal of Solids and Structures 42 (26), 6628-6642.

URL http://linkinghub.elsevier.com/retrieve/pii/ S0020768305001137

Geslain, A., Dazel, O., Groby, J. P., Sahraoui, S., Lauriks, W., aug 2011. Influence of static compression on mechanical parameters of acoustic foams. The Journal of the Acoustical Society of America 130 (2), 818-25.

URL http://www.ncbi.nlm.nih.gov/pubmed/21877797

Gibson, L. J., Ashby, M. F., 1999. Cellular Solids: Structure And Properties. Cambridge University Press.

Gong, L., Kyriakides, S., Jang, W.-Y., mar 2005. Compressive response of open-cell foams. Part I: Morphology and elastic properties. International Journal of Solids and Structures 42 (5-6), 1355-1379.

URL http://linkinghub.elsevier.com/retrieve/pii/ S0020768304004305

Göransson, P., jan 2006. Acoustic and vibrational damping in porous solids. Philosophical transactions. Series A, Mathematical, physical, and engineering sciences 364 (1838), 89-108.

URL http://www.ncbi.nlm.nih.gov/pubmed/18272454

Hoang, M. T., Bonnet, G., Tuan Luu, H., Perrot, C., 2014. Linear elastic properties derivation from microstructures representative of transport parameters. The Journal of the Acoustical Society of America 135 (June 
2014), 3172.

URL http://www .ncbi.nlm.nih.gov/pubmed/24907783

Jang, W.-y., Hsieh, W.-y., Miao, C.-c., Yen, Y.-c., 2015. Microstructure and mechanical properties of ALPORAS closed-cell aluminium foam. Materials Characterization 107, 228-238.

URL http://dx.doi.org/10.1016/j.matchar.2015.07.012http: //wWw.sciencedirect.com/science/article/pii/S1044580315002594

Jang, W.-Y., Kraynik, A. M., Kyriakides, S., apr 2008. On the microstructure of open-cell foams and its effect on elastic properties. International Journal of Solids and Structures 45 (7-8), 1845-1875.

URL http://linkinghub.elsevier.com/retrieve/pii/ S0020768307004118

Jang, W.-Y., Kyriakides, S., Kraynik, A. M., oct 2010. On the compressive strength of open-cell metal foams with Kelvin and random cell structures. International Journal of Solids and Structures 47 (21), 2872-2883.

URL http://linkinghub.elsevier.com/retrieve/pii/ S0020768310002271

Johnson, D. L., Koplik, J., Dashen, R., 1987. Theory of dynamic permeability and tortuosity in fluid-saturated porous media. Journal of Fluid Mechanics $176,379-402$.

Kanaun, S., Tkachenko, O., 2007. Representative volume element and effective elastic properties of open cell foam materials with random microstructures. Journal of Mechanics of Materials and Structures 2 (October), 1607- 
1628.

URL http://msp.org/jomms/2007/2-8/jomms-v2-n8-p17-s.pdf

Kino, N., Ueno, T., apr 2008. Comparisons between characteristic lengths and fibre equivalent diameters in glass fibre and melamine foam materials of similar flow resistivity. Applied Acoustics 69 (4), 325-331.

URL http://linkinghub.elsevier.com/retrieve/pii/ S0003682X06002325

Langlois, C., Panneton, R., Atalla, N., 2001. Polynomial relations for quasi-static mechanical characterization of isotropic poroelastic materials. The Journal of the Acoustical Society of America 110 (6), 3032.

URL http://link.aip.org/link/JASMAN/v110/i6/p3032/s1\{\&\}Agg= doi

Li, K., Gao, X.-L., a.K. Roy, sep 2003. Micromechanics model for threedimensional open-cell foams using a tetrakaidecahedral unit cell and Castigliano's second theorem. Composites Science and Technology 63 (12), 1769-1781.

URL http://linkinghub.elsevier.com/retrieve/pii/ S0266353803001179

Li, K., Gao, X.-L., Subhash, G., apr 2006. Effects of cell shape and strut cross-sectional area variations on the elastic properties of threedimensional open-cell foams. Journal of the Mechanics and Physics of Solids 54 (4), 783-806.

URL http://linkinghub.elsevier.com/retrieve/pii/ S0022509605002048 
Lu, Z.-X., Liu, Q., Huang, J.-X., dec 2011. Analysis of defects on the compressive behaviors of open-cell metal foams through models using the FEM. Materials Science and Engineering: A 530, 285-296.

URL http://linkinghub.elsevier.com/retrieve/pii/ S0921509311010598

Maheo, L., Viot, P., Bernard, D., Chirazi, A., Ceglia, G., Schmitt, V., Mondain-Monval, O., jan 2013. Elastic behavior of multi-scale, open-cell foams. Composites Part B: Engineering 44 (1), 172-183.

URL http://linkinghub.elsevier.com/retrieve/pii/ S1359836812003897

Mills, N., jan 2007. The high strain mechanical response of the wet Kelvin model for open-cell foams. International Journal of Solids and Structures 44 (1), 51-65.

URL http://linkinghub.elsevier.com/retrieve/pii/ S0020768306001235

Perrot, C., Chevillotte, F., Panneton, R., aug 2008. Bottom-up approach for microstructure optimization of sound absorbing materials. The Journal of the Acoustical Society of America 124 (2), 940-948.

URL http://www.ncbi.nlm.nih.gov/pubmed/18681586

Perrot, C., Panneton, R., Olny, X., 2007a. Computation of the dynamic thermal dissipation properties of porous media by Brownian motion simulation: Application to an open-cell aluminum foam. Journal of Applied Physics 102 (7), 074917. 
URL http://scitation.aip.org/content/aip/journal/jap/102/7/ $10.1063 / 1.2786899$

Perrot, C., Panneton, R., Olny, X., 2007b. Periodic unit cell reconstruction of porous media: Application to open-cell aluminum foams. Journal of Applied Physics 101 (11), 113538.

URL http://scitation.aip.org/content/aip/journal/jap/101/11/ $10.1063 / 1.2745095$

Redenbach, C., Shklyar, I., Andrä, H., 2012. Laguerre tessellations for elastic stiffness simulations of closed foams with strongly varying cell sizes. International Journal of Engineering Science 50 (1), 70-78.

URL http://dx.doi.org/10.1016/j.ijengsci.2011.09.002

Ridha, M., Shim, V. P. W., apr 2008. Microstructure and Tensile Mechanical Properties of Anisotropic Rigid Polyurethane Foam. Experimental Mechanics 48 (6), 763-776.

URL http://link.springer.com/10.1007/s11340-008-9146-0

Roberts, A. P., Garboczi, E. J., 2001. Elastic Moduli of Model Random Three- Dimensional Closed-Cell Cellular Solids. Acta mater 49 (2), 189197.

URL wWw.elsevier . com/locate/actamat

Salissou, Y., Panneton, R., 2007. Pressure/mass method to measure open porosity of porous solids. Journal of Applied Physics 101 (12), 124913124913-7. 
URL http://scitation.aip.org/content/aip/journal/jap/101/12/ $10.1063 / 1.2749486$

Shivakumar, N., Deba, A., Chaudhary, A., 2011. An Experimental Study on Mechanical Behavior and Microstructures of Polyurethane Foams for Design Applications. International Journal of ... 3 (3), 163-169.

URL http://multi-science.metapress.com/index/ H37479U32401J7K3.pdf

Storm, J., Abendroth, M., Kuna, M., 2015. Influence of curved struts, anisotropic pores and strut cavities on the effective elastic properties of open-cell foams. Mechanics of Materials 86, 1-10.

URL http://dx.doi.org/10.1016/j.mechmat.2015.02.012

Sullivan, R. M., Ghosn, L. J., oct 2009. Shear moduli for non-isotropic, open cell foams using a general elongated Kelvin foam model. International Journal of Engineering Science 47 (10), 990-1001.

URL http://linkinghub.elsevier.com/retrieve/pii/ S0020722509000767

Sullivan, R. M., Ghosn, L. J., Lerch, B. a., mar 2008. A general tetrakaidecahedron model for open-celled foams. International Journal of Solids and Structures 45 (6), 1754-1765.

URL http://linkinghub.elsevier.com/retrieve/pii/ S0020768307004465

Thiyagasundaram, P., Sankar, B. V., Arakere, N. K., apr 2010. Elastic Properties of Open-Cell Foams with Tetrakaidecahedral Cells Using Finite El- 
ement Analysis. AIAA Journal 48 (4), 818-828.

URL http://arc.aiaa.org/doi/abs/10.2514/1 . J050022

Thomson, W., 1887. On the division of space with minimum partitional area. Acta Mathematica 11, 121- 134.

URL http://link.springer.com/article/10.1007/BF02612322

Veyhl, C., Belova, I., Murch, G., Fiedler, T., may 2011. Finite element analysis of the mechanical properties of cellular aluminium based on micro-computed tomography. Materials Science and Engineering: A 528 (13-14), 4550-4555.

URL http://linkinghub.elsevier.com/retrieve/pii/ S092150931100195X

Xia, Z., Zhou, C., Yong, Q., Wang, X., jan 2006. On selection of repeated unit cell model and application of unified periodic boundary conditions in micro-mechanical analysis of composites. International Journal of Solids and Structures 43 (2), 266-278.

URL http://linkinghub.elsevier.com/retrieve/pii/ S0020768305001460

Zhang, C., Li, J., Hu, Z., Zhu, F., Huang, Y., 2012. Correlation between the acoustic and porous cell morphology of polyurethane foam: Effect of interconnected porosity. Materials and Design 41, 319-325.

URL http://dx.doi.org/10.1016/j.matdes.2012.04.031

Zhou, J., Mercer, C., Soboyejo, W., 2002. An investigation of the microstructure and strength of open-cell 6101 aluminum foams. Metallurgical and 
Materials Transactions ... 33A (May), 1413-1427.

URL http://link.springer.com/article/10.1007/

s11661-002-0065-x

Zhu, H., Hobdell, J., a.H Windle, dec 2000. Effects of cell irregularity on the elastic properties of open-cell foams. Acta Materialia 48 (20), 4893-4900.

URL http://linkinghub.elsevier.com/retrieve/pii/ S1359645400002822

Zhu, H., Knott, J., Mills, N., mar 1997. Analysis of the elastic properties of open-cell foams with tetrakaidecahedral cells. Journal of the Mechanics and Physics of Solids 45 (3), 319-343.

URL

http://linkinghub.elsevier.com/retrieve/pii/

S0022509696000907 